\title{
Solvent-assisted dye-diffusion thermal transfer for electronic imaging applications
}

\author{
Chung-Chih $\mathrm{Wu}^{\text {a) }}$ Sun-Wen Lin, Chieh-Wei Chen, and Jui-Hung Hsu \\ Department of Electrical Engineering, Graduate Institute of Electro-Optical Engineering, \\ and Graduate Institute of Electronics Engineering, National Taiwan University, Taipei, Taiwan 10617, \\ Republic of China
}

(Received 22 January 2001; accepted for publication 5 December 2001)

\begin{abstract}
Molecularly doped polymers processed by dye-diffusion thermal transfer (D2T2) have wide applications in electronic imaging. For the sake of stability, it is usually desired that the host polymers have high glass-transition temperatures $\left(T_{g}\right)$, which however implies high processing temperatures. In this letter, we show that in an atmosphere of organic solvent vapor, effective dye-diffusion thermal transfer may be carried out at temperatures much below the $T_{g}$ of a host polymer. The atmosphere of solvent vapor ensures incorporation of solvent molecules into the polymer throughout the process and causes the plasticization effect. As a consequence, the effective $T_{g}$ of the system is lowered and the diffusion of dyes in the host polymer is enhanced. Through such a process, polymers of higher $T_{g}$ and wider ranges of dye molecules may be used in D2T2 without losing the processing compatibility. Furthermore, carrying out D2T2 at lower temperatures is beneficial to the resolutions of transferred features due to the suppression of vaporization of dyes. (C) 2002 American Institute of Physics. [DOI: 10.1063/1.1447001]
\end{abstract}

Dye-diffusion thermal transfer (D2T2) printing, is an electronic printing process having wide applications in electronic imaging, such as in making color prints and color filters. ${ }^{1}$ In D2T2 printing, image information in the form of electronic signals is converted to a pattern of thermal energy by an array of heating elements or a laser beam creating a corresponding pattern of increased temperature in the ribbon and the thermal transfer paper in intimate contact. Consequently, dyes in the donor polymer layer on the ribbon transfer by diffusion into the receiver polymer layer on the thermal transfer paper. Recently, such a D2T2 process has also been applied to another form of electronic imaging, i.e., the organic light-emitting devices or displays (OLEDs), for adding emissive dopant molecules into luminescent organic host films for color tuning or efficiency enhancement. ${ }^{2-4}$ Such doping processes have been used to produce lateral dye patterns in polymer films for color integration in OLEDs. ${ }^{2,3}$ In addition, the capability of D2T2 to produce graded doping profiles has been used to confine the vertical dopant distribution around the carrier recombination zone of OLED devices, such that the effect of carrier trapping due to emissive dopants can be minimized in devices incorporating molecularly doped polymers. ${ }^{4}$

To obtain practical transfer rates, the D2T2 process is usually performed at temperatures around or above the $T_{g}$ of glassy host polymers. ${ }^{1}$ Above $T_{g}$, the polymers lose glasslike properties, assume those more commonly identified with a rubber, and are accompanied by more segmental motion of polymer chains. Consequently, the space between polymer chains (i.e., the free volume) increases as usually indicated by the rapid increase of specific volume above $T_{g} \cdot{ }^{5}$ Hence

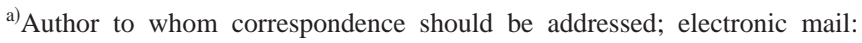
chungwu@cc.ee.ntu.edu.tw
}

dye molecules can diffuse much faster above $T_{g}$ than below $T_{g}$. From the considerations of stability, it is desired that dopant molecules diffused at elevated temperatures be free from migration within the nominal temperature range of applications. To "freeze" dopant molecules at operation temperatures, it usually requires that the receiver polymer owns a high enough $T_{g}$ and that the diffusion capability of chosen dye molecules drops rapidly with temperature. ${ }^{1,3,4}$ Such conditions, however, imply high processing temperatures for acceptable transfer rates and impose a limitation on the availability of dopant molecules due to considerations of thermal stability. In this letter, we report a solvent-assisted process permitting effective D2T2 to be carried out at temperatures much below $T_{g}$ of the host polymers. Thus, without losing the processing compatibility, polymers of even higher $T_{g}$ and wider ranges of dye molecules may be used in the D2T2 process for electronic imaging applications.

It has been known that the $T_{g}$ of glassy polymers can be reduced with the presence of additives, such as the plasticizers of low molecular weight. ${ }^{5,6}$ In applications sensitive to the presence of additives ("impurities"), it is desired that the plasticizers can be removed after the process. Organic solvent molecules represent a good choice in such sense, because they can be absorbed into a polymer for reducing $T_{g}$ and be evaporated afterward. Based on the free-volume considerations, the effect of plasticization on $T_{g_{5}}$ can be approximately estimated by the following equation: ${ }^{5}$

$$
T_{g} \approx \frac{T_{g p}}{1+\left(\frac{3 T_{g p}}{2 T_{m s}}-1\right)\left(1-\Phi_{p}\right)},
$$

where $T_{g p}$ is the glass transition temperature of the pure polymer, $T_{m s}$ is the melting point of the solvent, and $\Phi_{p}$ and $\left(1-\Phi_{p}\right)$ are the volume fractions of the polymer and the 


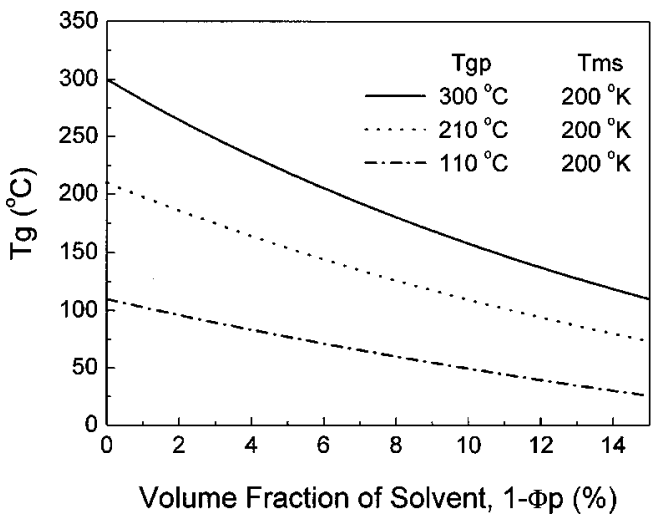

FIG. 1. $T_{g}$ vs the volume fraction of the solvent $\left(1-\Phi_{p}\right)$, for various $T_{g p}$ and $T_{m s}$.

solvent, respectively. To evaluate the effect of solvent incorporation on the effective $T_{g}$, Fig. 1 shows $T_{g}$ as a function of $\left(1-\Phi_{p}\right)$ for various $T_{g p}$ 's and for a typical $T_{m s}$ of $200^{\circ} \mathrm{K}$. It indicates that the presence of a small fraction of solvent in the polymer can result in a significant reduction of $T_{g}$, and that the D2T2 below $T_{g p}$ may be facilitated through incorporation of solvent molecules.

The experiment setup for performing the solvent-assisted D2T2 is shown in Fig. 2. In a chamber purged with pure $\mathrm{N}_{2}$, a beaker containing the organic solvent was heated to provide the solvent vapor. Under such an atmosphere, the finitesource dye-diffusion thermal transfer (FS-D2T2) ${ }^{3,4}$ was carried out on another temperature-controlled stage. In the FSD2T2 process, a neat receiver polymer layer on one plate was placed in direct contact with the dye-dispersed donor polymer layer on another plate to permit direct D2T2 at elevated temperatures. Prior to the FS-D2T2, both the source and receiver films were let absorb the solvent vapor. Initial experiments that samples were treated by solvent vapor but the actual D2T2 was carried out in the ambient atmosphere with no solvent vapor exhibited very limited enhancement of the D2T2 transfer rate. It is mainly because the solvent molecules absorbed into the polymer films were rapidly evaporated at elevated temperatures. Thus, the whole D2T2 process was eventually performed in the vapor atmosphere to ensure incorporation of solvent molecules throughout the process. The progress of the FS-D2T2 process and the ratio of dyes transferred were monitored by the absorption intensities of dyes into the receiver films. Previous theoretical and

\section{Solvent-assisted D2T2 Process Chamber}

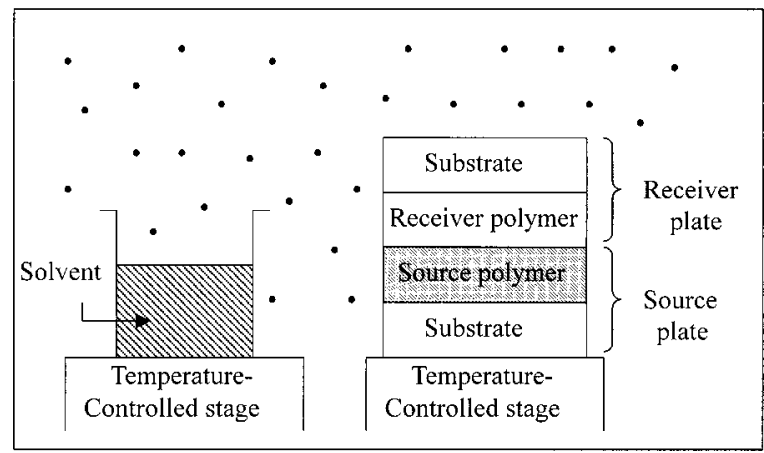

FIG. 2. Experimental setup for performing the solvent-assisted D2T2.

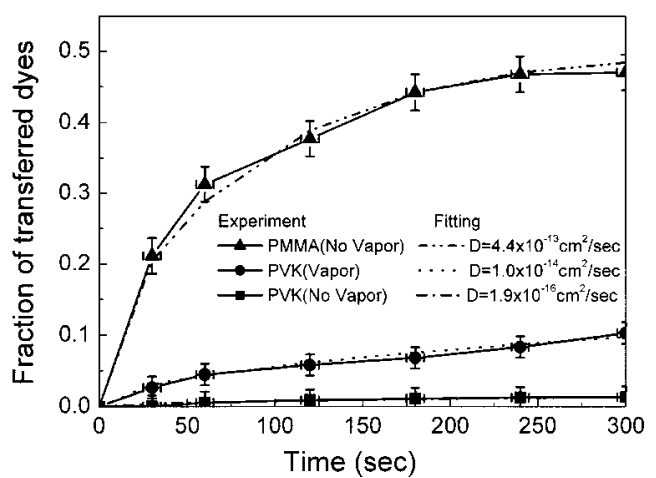

(a)

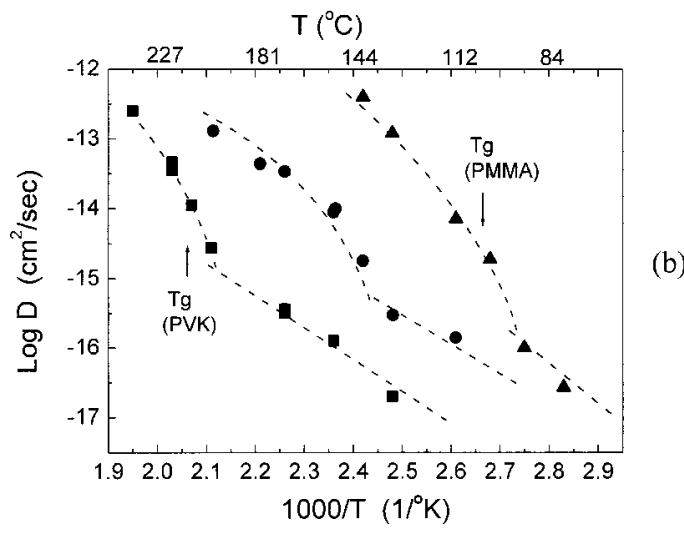

FIG. 3. (a) Ratios of dyes transferred vs diffusion time at $150{ }^{\circ} \mathrm{C}$ for three different cases; (b) extracted diffusion coefficients of the dye vs reciprocal temperature in different matrices. Dashed lines in (b) are just for eye guiding.

experimental studies indicate that the FS-D2T2 process can be modeled by Fick's diffusion theory under impermeable film-substrate boundary conditions. ${ }^{3,4}$

Two glassy polymers studied were $\operatorname{poly}(\mathrm{N}-$ vinylcarbazole) (PVK) and poly(methyl methacrylate) (PMMA). PVK is a thermally stable, hole-transport and deep-blue emitting polymer often used in OLEDs, ${ }^{7-10}$ and PMMA is a transparent and inert polymer. The differential scanning calorimetry (DSC) of the PVK and PMMA samples show distinct glass transitions around 210 and $110^{\circ} \mathrm{C}$, respectively. In the FS-D2T2 experiments, both the source and the receiver layers use the same polymer and the same layer thickness of $\sim 1000 \AA$. Coumarin 6 (C6, green) was used as dye dopants with an initial concentration of $3 \mathrm{wt} \%$ in the source polymer. Acetone was used as the solvent for its relatively high vapor pressure $(\sim 160 \mathrm{mmHg}$ at room temperature) and its low melting point $\left(200{ }^{\circ} \mathrm{K}\right)$.

Figure 3(a) shows experimentally determined ratios of dyes transferred versus diffusion time at $150{ }^{\circ} \mathrm{C}$ for three different cases: (i) PVK with no solvent vapor, (ii) PMMA with no solvent vapor, and (iii) PVK with acetone vapor. The transfer in PMMA is rapid and the equilibrium is nearly reached in a short time, since this temperature is substantially higher than its $T_{g}$. In sharp contrast, the transfer in PVK with no solvent vapor is extremely slow since this temperature is substantially lower than $T_{g}$ of PVK. However, the transfer rate in PVK has been largely enhanced with the presence of acetone vapor. The effects of $T_{g}$ and solvent vapor on diffusion can be further explicated in Fig. 3(b), which shows diffusion coefficients of C6 versus the reciprocal diffusion temperature $(D-1 / T$ curve) in different matrices. $D$ at 


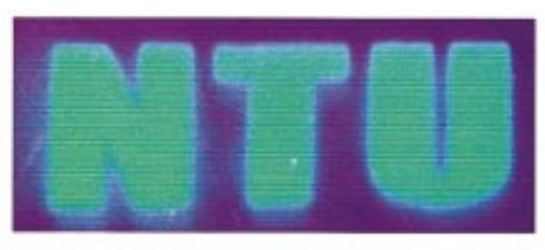

(a)

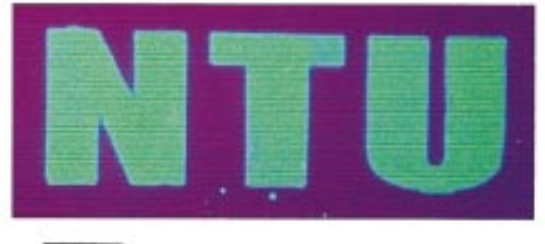

(b)

$2 \mathrm{~mm}$

FIG. 4. (Color) PL images of (a) a C6 pattern (green) in PVK (deep blue) transferred by conventional D2T2 at $220^{\circ} \mathrm{C}$, and (b) a C6 pattern in PVK transferred by solvent-assisted D2T2 at $180^{\circ} \mathrm{C}$.

a diffusion temperature is extracted from the least-square fitting of the experimentally measured ratios of dyes transferred versus diffusion time by Fick's diffusion theory with $D$ as the only parameter, as illustrated in Fig. 3(a). As seen in Fig. 3(b), the temperature dependence of $D$ exhibits a transition around $T_{g}$ for both polymers, typical for dye diffusion in glassy polymers, and the magnitudes of the diffusion coefficients around $T_{g}$ are consistent with those found in previous studies. ${ }^{11-14}$ What is the most profound is the shift of PVK's $D-1 / T$ curve to lower temperatures with the presence of solvent vapor. The shift is not parallel, however, with smaller shifts at larger values of $D$. It is presumably because the incorporation of solvent molecules into the polymer films drops as film temperature increases, leading to less reduction of $T_{g}$. At smaller values of $D$, a shift of $\sim 50-70{ }^{\circ} \mathrm{C}$ is observed, suggesting of the lowering of $T_{g}$ by a similar value. According to Eq. (1) and Fig. 1, such a lowering of $T_{g}$ represents a volume fraction of about $4 \%$ for acetone molecules in PVK. It is qualitatively consistent with the difference in thickness between a solvent-vapor-treated and a thoroughly dried PVK films, as determined by ellipsometry.

The capability to carry out D2T2 at lower temperatures for a high- $T_{g}$ polymer may be beneficial to the resolution of transferred dye patterns. Figure 4(a) shows an UV-excited photoluminescence (PL) image of a C6 pattern (green) in a PVK receiver film (deep-blue), transferred from a patterned PVK source film by conventional D2T2 $\left(220^{\circ} \mathrm{C}, 1 \mathrm{~min}\right)$. Clearly, it is seen that the pattern is blurred with nonintended doping occurring around the edges of the pattern. It is presumably due to the evaporation of dyes out of the edges of either donor or receiver patterns during high-temperature D2T2, followed by diffusion into nearby areas of the receiver film. By performing D2T2 at lower temperatures with the solvent-assisted process, the vaporization of dyes and related problems can be effectively suppressed. As a result, the resolution of transferred patterns is greater, as illustrated in Fig. 4(b), which shows a PL image of a C6 pattern in PVK transferred by solvent-assisted D2T2 $\left(180^{\circ} \mathrm{C}\right)$ under otherwise similar conditions.

While preceding results clearly demonstrate the feasibility of the present method we are aware that the optimization of a few factors may further improve this process. First, one may increase the incorporation of solvent molecules in the polymer by raising the vapor pressure of the solvent. In the present cold-wall chamber, the vapor pressure of the solvent is, to first order, its saturated vapor pressure at the room temperature. Replacing the process chamber with a hot-wall one, the atmosphere temperature may permit a higher vapor pressure without causing condensation of the solvent. Second, using solvents with solubility parameters better matching that of the host polymer may enhance the polymersolvent compatibility and therefore incorporation of solvents molecules. ${ }^{5}$ Finally, as suggested by Eq. (1), using solvents of even lower melting points could also be helpful for further reduction of $T_{g}$.

In conclusion, we have shown that in an atmosphere of organic solvent vapor, effective dye-diffusion thermal transfer may be carried out at temperatures much below $T_{g}$ of host glassy polymers. Such an effect may permit polymers of higher $T_{g}$ and wider ranges of dye molecules to be used in the D2T2 process for electronic imaging applications. Carrying out D2T2 at lower temperatures is beneficial to the resolutions of transferred features due to the suppression of vaporization of dyes.

The authors are grateful to Professor T. Y. Luh, Professor M. K. Leung of NTU and Dr. W. K. Weng of MRL/ITRI for stimulating discussions and for assistance in experiments. This work was supported by National Science Council (Grant No. NSC 89-2215-E-002-025) and Ministry of Education of the R.O.C.

${ }^{1}$ Chemical Technology in Printing and Imaging Systems, edited by J. A. G. Drake (Royal Society of Chemistry, Cambridge, UK, 1993).

${ }^{2}$ F. Pschenitzka and J. C. Sturm, Appl. Phys. Lett. 74, 1913 (1999).

${ }^{3}$ C.-C. Wu, C.-C. Yang, H.-H. Chang, C.-W. Chen, and C.-C. Lee, Appl. Phys. Lett. 77, 794 (2000).

${ }^{4}$ H.-H. Chang, C.-C. Wu, C.-C. Yang, C.-W. Chen, and C.-C. Lee, Appl. Phys. Lett. 78, 574 (2001).

${ }^{5}$ J. Bicerano, Prediction of Polymer Properties, 2nd ed. (Marcel Dekker, New York, 1996).

${ }^{6}$ D. Y. Khang and H. H. Lee, Appl. Phys. Lett. 76, 870 (2000).

${ }^{7}$ W. D. Gill, J. Appl. Phys. 43, 5033 (1972).

${ }^{8}$ R. H. Partridge, Polymer 24, 748 (1983).

${ }^{9}$ J. Kido, K. Hongawa, K. Okuyama, and K. Nagai, Appl. Phys. Lett. 63, 2627 (1993).

${ }^{10}$ C. C. Wu, J. C. Sturm, R. A. Register, J. Tian, E. P. Dana, and M. E. Thompson, IEEE Trans. Electron Devices ED-44, 1269 (1997).

${ }^{11}$ D. Ehlich and H. Sillescu, Macromolecules 23, 1600 (1990).

${ }^{12}$ P. F. Nealey, R. E. Cohen, and A. S. Argon, Macromolecules 26, 1287 (1993).

${ }^{13}$ J. M. Zielinski, G. Heuberger, H. Sillescu, U. Wiesner, A. Heuer, Y. Zhang, and H. W. Spiess, Macromolecules 28, 8287 (1995).

${ }^{14}$ D. D. Deppe, A. Dhinojwala, and J. M. Torkelson, Macromolecules 29, 3898 (1996) 
Applied Physics Letters is copyrighted by the American Institute of Physics (AIP).

Redistribution of journal material is subject to the AIP online journal license and/or AIP copyright. For more information, see http:/ojps.aip.org/aplo/aplcr.jsp

Copyright of Applied Physics Letters is the property of American Institute of Physics and its content may not be copied or emailed to multiple sites or posted to a listserv without the copyright holder's express written permission. However, users may print, download, or email articles for individual use. 